\title{
An approach for constraining mantle viscosities through assimilation of paleo sea level data into a glacial isostatic adjustment model
}

\author{
Reyko Schachtschneider ${ }^{1}$, Jan Saynisch-Wagner ${ }^{1}$, Volker Klemann ${ }^{1}$, Meike Bagge ${ }^{1}$, and Maik Thomas ${ }^{1,2}$ \\ ${ }^{1}$ Helmholtz Centre Potsdam GFZ German Research Centre for Geosciences, Telegrafenberg, 14473 Potsdam, Germany \\ ${ }^{2}$ Freie Universität Berlin, Kaiserswerther Str. 16-18, 14195 Berlin, Germany
}

Correspondence: Reyko Schachtschneider (reyko.schachtschneider@gfz-potsdam.de)

\begin{abstract}
.
Glacial isostatic adjustment is largely governed by rheological properties of the Earth's mantle. Large mass redistributions in the ocean-cryosphere system and the subsequent response of the visco-elastic Earth have led to dramatic sea level changes in the past. This process is ongoing and in order to understand and predict current and future sea level changes the knowledge of mantle properties such as viscosity is essential. In this study we present a method to obtain estimates of mantle viscosities by assimilation of relative sea level data into a visco-elastic model of the lithosphere and mantle. We set up a particle filter with probabilistic resampling. In an identical twin experiment we show that mantle viscosities can be recovered in a glacial isostatic adjustment model of a simple three layer earth structure consisting of an elastic lithosphere and two mantle layers of different viscosity. In two scenarios we investigate the dependence of the ensemble behavior on the ensemble initialization and observation uncertainties and show that the recovery is successful if the target parameter values are properly sampled by the initial ensemble probability distribution. This even includes cases in which the target viscosity values are located far in the tail of the initial ensemble probability distribution. We then successfully apply the method to two special cases that are relevant for the assimilation of real observations: 1) using observations taken from a single region only, here Laurentide and Fennoscandia, respectively, and 2) using only observations from the last 10 kyrs.
\end{abstract}

\section{Introduction}

Glacial isostatic adjustment (GIA) describes the continual response of the Earth to mass redistribution between continental glaciers, ice sheets and the ocean during glacial cycles (e.g., Lambeck et al., 2003). These quasi-periodic mass redistributions occur due to climate cycles that have their origin in astronomical cycles of precession, obliquity, and eccentricity with periods near 23,000, 41,000, and 96,000 years (Imbrie et al., 1992). In the past, deformation of the Earth's surface due to those mass redistributions have led to raising and falling sea levels with local amplitudes exceeding a hundred meters (e.g. Haskell, 1935; Lambeck et al., 1998; Whitehouse, 2018).

Understanding GIA processes is essential for the quantification of past and recent sea level changes. Especially, the rheology of the Earth's mantle plays a significant role in surface deformation (Lambeck et al., 1998). Therefore, obtaining reliable 
values for mantle viscosity is the basis for a precise determination of Earth's deformation history, mass re-distribution, and sea level changes. Especially, when trying to estimate the distribution of sea level change due to ongoing melting of glaciers and continental ice sheets in Greenland and Antarctica, the precise knowledge of isostatic adjustment processes is indispensable.

Since GIA processes are mainly governed by mantle viscosity, there have been numerous studies attempting to estimate viscosity values and its depth distributions. The Earth's response after deglaciation is one important process that allows to infer mantle viscosities (Peltier, 1996; Steffen and Wu, 2011).

In this study, we demonstrate a method that allows to draw conclusions about mantle viscosity values by assimilating relative sea level (RSL) observations into a visco-elastic Earth model. In an identical twin experiment we assimilate RSL rates computed from a reference model. We apply a particle filter and study how the mean parameter state of the model ensemble converges to the target parameter state. First, we demonstrate the applicability of the method and then show two special cases that are relevant for assimilation of real RSL observations.

The paper is organized as follows. In section 2 the visco-elastic deformation model is described. Section 3 deals with the basic principles of data assimilation and the particular filter that was used in this study. The experiment setup description follows in Sect. 4. Our results are presented and discussed in Sect. 5 and Sect. 6, respectively, followed by some concluding remarks and an outlook in Sect. 7.

\section{Glacial Isostatic Adjustment Model}

In this study, we facilitate the VIsco-elastic model of the Lithosphere and MAntle (VILMA) (Klemann et al., 2008). In VILMA, the problem of surface deformation of a self-gravitating, visco-elastic and incompressible Earth is solved in the time domain following the spectral-finite element (SFE) formulation of Martinec (2000). The model is capable of handling 3D viscosity distributions (Klemann et al., 2008) and considers the water-mass redistribution between ice-sheets and ocean gravitationally consistently the way it was proposed by Farrell and Clark (1976) considering moving coast lines and floating ice (Hagedoorn et al., 2007). The solution in the time domain allows a direct update of the visco-elastic model state during the assimilation process. This is described in more detail in Sect. 3.

Here, we consider a 1D Earth structure, i.e. viscosity varies only with depth, and consider the viscosity to be constant in the lower and upper mantle. The transition from lower to upper mantle is at $670 \mathrm{~km}$ depth. The lithosphere is considered as an elastic region of $60 \mathrm{~km}$ thickness at the top, and the fluid core is considered as a lower boundary condition. Shear modulus and density follows the elastic PREM structure (Dziewonski and Anderson, 1981). The radial finite elements' thicknesses are ranging between $40 \mathrm{~km}$ at the base of the lower mantle to $5 \mathrm{~km}$ in the lithosphere and sum up in total to 164 SFEs in the vertical (cf. Table 1). In horizontal directions the problem is solved in spherical harmonics, degree and order ranging from 0 to 170. The model parameters are discretized on a $256 \times 512$ grid.

As forcing, the surface mass load of the last glacial cycle in the parameterization of the ICE-5G reconstruction (Peltier, 2004) is considered. In time, this process covers the time range from $123 \mathrm{ka}$ BP to present. The integration time step in the dynamic model was set to 20 years. 
Table 1. Depth structure of VILMA model in this study with number of spectral-finite elements (SFEs) in vertical direction per region and viscosity of the reference model.

\begin{tabular}{lccc}
\hline Region & Depth [km] & Number of SFEs & Viscosity [Pa s] \\
\hline Lithosphere & $0-60$ & 12 & $10^{30}$ \\
Upper mantle & $60-670$ & 97 & $10^{20}$ \\
Lower mantle & $670-3,891$ & 55 & $10^{21}$ \\
\hline
\end{tabular}

\section{Data Assimilation}

\subsection{General}

Data assimilation provides a way to combine dynamic models with observations (Asch et al., 2016). Using data assimilation techniques, a model can be updated based on observations in order to obtain new model parameters that better explain the data. There are various data assimilation techniques that are appropriate for certain applications or scenarios. They have been used in a wide range of scientific fields, including numerical weather prediction (Bauer et al., 2015), ocean circulation modelling (Saynisch et al., 2015; Irrgang et al., 2017), geomagnetic field modeling (Bärenzung et al., 2018) and geodynamo studies (Fournier et al., 2013).

A well-known method for solving non-linear filtering problems with non-Gaussian error statistics is the extended Kalman filter by Anderson and Moore (1979). Its principle is based on linearization of evolution models with Taylor series expansions. Such approximation can lead to poor representations of the models non-linearities and its probability density function (PDF) and the filter can diverge (Van der Merwe et al., 2001).

In our study, we used the particle filter for parameter estimation in the dynamic model VILMA. The goal of parameter estimation is to find a set of parameters in a model that leads to a solution that is consistent with a set of observations (Evensen, 2009). The parameters we attempt to estimate are the viscosities of the lower and upper mantle.

\subsection{The particle filter}

The particle filter is an ensemble-based data assimilation technique. It follows the Monte-Carlo view that any probability distribution can be represented by a discrete sample from that distribution (Liu et al., 2001). Ensemble based techniques are particularly useful if the models are non-linear and the PDF of the model state or the errors are not Gaussian (Van Leeuwen, 2009). It allows a complete representation of the posterior model state distribution so that statistical estimates are easy to compute (Van der Merwe et al., 2001). In a particle filter, each ensemble member is assigned a weight factor which is updated during each assimilation step. As in basic particle filters, the ensemble members did not mix they were called particles. The output of the filter is the weighted mean of the model state. In particle filters without resampling, after some assimilation steps the ensemble can contain particles with very low weights that are practically insignificant (Pham, 2001). Therefore, without resampling large ensembles are needed in order to properly sample the model PDF. If too few particles with significant 
weight remain, the filter is degenerated and does not represent the model PDF. Therefore, if particles with low significance are resampled to particles with higher significance, the ensemble size can be reduced while the ensemble PDF can still represent the model PDF. More profound introductions to data assimilation and particle filters can be found, e.g., in Asch et al. (2016), Carrassi et al. (2018), and Fearnhead and Künsch (2018).

The model update in our particle filter is based directly on Bayes' theorem (for an introduction see e.g. (Box and Tiao, 2011). If the PDFs of the model and the PDFs of the observations are continuous, Bayes' theorem holds:

$p_{\mathrm{m}}(\boldsymbol{\psi} \mid \boldsymbol{d})=\frac{p_{\mathrm{d}}(\boldsymbol{d} \mid \boldsymbol{\psi}) p_{\mathrm{m}}(\boldsymbol{\psi})}{p_{\mathrm{d}}(\boldsymbol{d})}$,

where $p_{\mathrm{m}}(\boldsymbol{\psi} \mid \boldsymbol{d})$ is the posterior PDF of the model given the observations, $p_{\mathrm{d}}(\boldsymbol{d} \mid \boldsymbol{\psi})$ is the likelihood of the observations given the model, $p_{\mathrm{m}}(\boldsymbol{\psi})$ is the prior PDF of the model, and $p_{\mathrm{d}}(\boldsymbol{d})$ a normalization factor, the so-called model evidence. The weights of a particle are given by

$w_{i}=\frac{p_{d}\left(\boldsymbol{d} \mid \boldsymbol{\psi}_{i}\right)}{\sum_{j=1}^{M} p_{d}\left(\boldsymbol{d} \mid \boldsymbol{\psi}_{j}\right)}$,

where $M$ is the ensemble size. Those weights represent the significance of a particle and its contribution to the estimate of the mean state.

We use a particle filter with importance resampling and perturbation. Its principle is illustrated in Fig. 1. Sequential Importance Resampling (SIR) was proposed by Rubin (1988) and applied to filtering of dynamical systems by Gordon et al. (1993). In this approach, an ensemble of model realizations is propagated in time. When observations become available, each ensemble member's performance is evaluated based on the differences between the observations and the corresponding values computed from the model states. These measures are used to decide which particles further propagate and which are disregarded after that assimilation step. Particles with low performance are resampled to states of better performing particles. Thus, the ensemble size stays constant throughout the entire assimilation run.

Resampling reduces the ensemble variance. If the variance becomes very small there is the risk of filter degeneracy (Fearnhead and Künsch, 2018), i.e. all ensemble members are resampled to only a few model states and the model PDF is no longer represented by the ensemble. There are three main approaches to overcome degeneracy (Fearnhead and Künsch, 2018): firstly, adding a random value to the estimated parameters (e.g. Liu and West, 2001); secondly, using a Monte-Carlo Markov Chain within the particle filter (Fearnhead, 2002) in order to obtain new parameter values; and thirdly, using a stochastic approximation method in which the particle filter update depends on the current parameter estimate (e.g. Poyiadjis et al., 2011). We follow the first approach since it is simple to implement and allows us to enhance the filter convergence by constraining the perturbation. While Liu and West (2001) proposed shrinking the ensemble to its mean state before adding noise, we add noise directly to the resampled particles. After resampling the particles, a random value based on the current ensemble variance is added to each particle's mantle viscosity values. The random values are drawn from scaled normal distributions $a N\left(0, \sigma_{\text {ens }}^{\mathrm{U}, \mathrm{L}}\right)$ for the two mantle regions separately, where $\sigma_{\text {ens }}^{\mathrm{L}}$ and $\sigma_{\text {ens }}^{\mathrm{U}}$ are the ensemble standard deviations (STD) for the lower and upper mantle, respectively. The scaling factor $a$ is introduced to control the convergence of the ensemble. It is set to 0.5 in this study. 


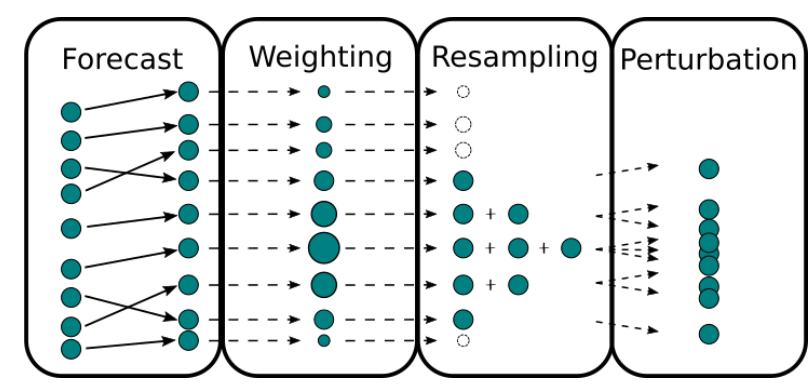

Figure 1. The particle filter principle. In the forecast phase the dynamic model ensemble is propagated in time until observations become available. Then, the ensemble members are assigned a weight factor based on the residuals between the observations and the according values computed from the model states. Based on the weight the ensemble is resampled. Members with low weights are disregarded, members with high weights are copied. The ensemble size stays constant. Finally, the model states of the ensemble members are perturbed and the next model integration cycle starts with the updated ensemble.

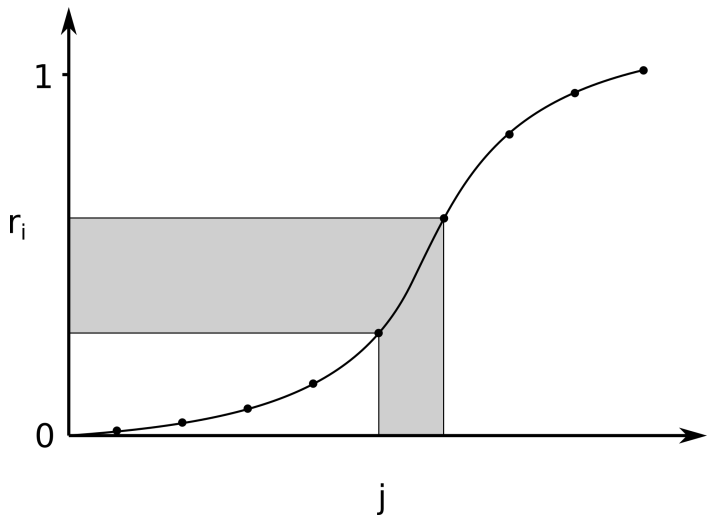

Figure 2. Resampling principle. After drawing a random number $r_{i}$ from a uniform distribution on [0,1] the $i$-th particle is resampled to the model state of the $j$-th particle if $r_{i}$ falls in the corresponding bin in the cumulative distribution of the normalized weights.

Figure 2 illustrates probabilistic resampling as used in our filter. After the forecast, the particle weights (cf. Eq. (2)) estimate how probable a particle is given the observations (Carrassi et al., 2018). For resampling, a value $w_{i}^{\prime}=\sum_{j=1}^{i} w_{j}$ is assigned to each particle. Then, $M$ random numbers $r_{i}$ are drawn from a uniform density on [0,1]. The $i$-th particle is resampled to the model state of the $j$-th particle if $w_{j-1}^{\prime}<r_{i} \leq w_{j}^{\prime}$.

For implementing the particle filter into the VILMA model the Parallel Data Assimilation Framework (PDAF) by Nerger et al. (2005) is used. It is a versatile software package that helps to include a variety of data assimilation techniques into pre-existing model codes. 


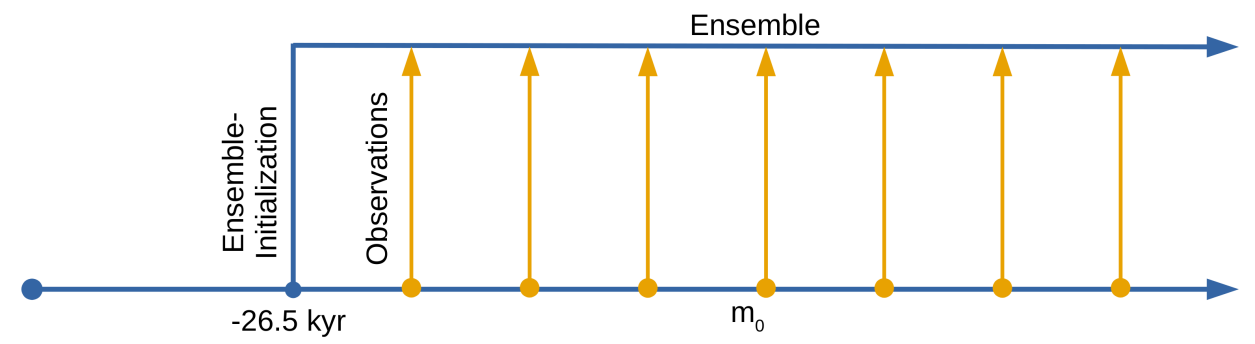

Figure 3. Setup of the identical twin experiment. The ensemble of particles is initialized from the model state of the target run $m_{0}$ at $t_{0}=-26.5 \mathrm{ka}$. During the assimilation steps, observations of RSL rates, obtained from the target run, are assimilated into the ensemble. This is done every 1 kyr.

\section{Experiment Setup}

\subsection{General}

The experiments we present are conducted as sandbox experiments. We use an identical twin setup for the reference model run and the assimilation simulation. Each ensemble is initialized from the model state of the reference run $m_{0}$ at time $t_{0}$. This is done by adding random values drawn from a normal distribution $N\left(\mu_{\text {init }}, \sigma_{\text {init }}\right)$ to the viscosity values $\nu$ of the lower and upper mantle, respectively, for each ensemble member. The reference run's mantle viscosity values function as target values for the assimilation experiments (cf. Table 1). All other model parameters remain unchanged. For the values governing the ensemble initialization, see Table 2. The RSL values determined by the reference model at respective locations and times are used to calculate the RSL rates that constitute the synthetic observations used in the assimilation.

In Fig. 3, the sandbox experiment principle is illustrated. From the initialization at $t_{0}$, the ensemble is propagated in time. At times $t_{n}=t_{0}+n \Delta t$, when synthetic observations are available, they are assimilated and the model ensemble is updated. The interval between consecutive observations is $\Delta t=1 \mathrm{kyr}$. Observations are continuously assimilated into the ensemble and the convergence of its weighted mean viscosity values (cf. Eq. (2)) to the values of the reference run, $m_{0}$, are investigated.

As a starting point for the assimilation we chose $t_{0}=26.5 \mathrm{ka} \mathrm{BP}$ and $10.5 \mathrm{ka} \mathrm{BP}$, respectively. The date $26.5 \mathrm{ka} \mathrm{BP}$ marks the beginning of the last glacial maximum (LGM). The date $10.5 \mathrm{ka} \mathrm{BP}$ approximately marks the end of the last deglaciation. Thereafter, the number of available observations increases significantly. Those setups are meaningful since the first setup gives large signals in RSL change while in the second setup more observations are available.

\subsection{Synthetic observations}

Geological sea level index points (SLIPs) allow to reconstruct former relative sea level. This is the water level, $S$, measured with respect to the earth surface, $T$, as well as referenced to present-day RSL.

$h_{\mathrm{rsl}}(t)=S(t)-T(t)-[S(t=0)-T(t=0)]$ 
Table 2. Parameters of the test cases investigated, with standard deviation of RSL observations $\left(\sigma_{\mathrm{obs}}\right)$, mean and standard deviation of ensemble initialization $\left(\mu_{\text {init }}\right.$ and $\left.\sigma_{\text {init }}\right)$, regions from which observations were used and the time interval of observations. Pairs of values represent lower / upper mantle viscosities.

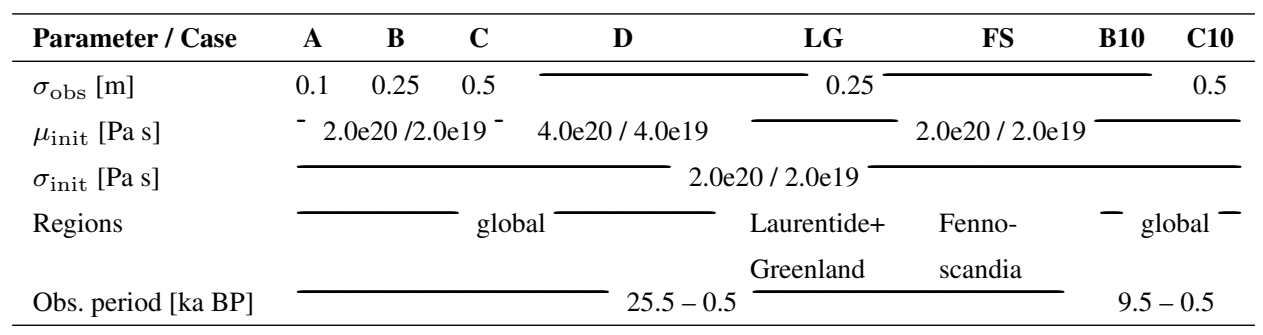

However, when modeling RSL in the forward sense considered here, $S(t)-T(t)$ is specified relative to the initial state. The referencing to $S(t=0)-T(t=0)$ can only be performed at the end of the integration, and, in consequence, its actual value will depend on the considered parameterization of the GIA process. Furthermore, for consideration of a realistic water-ice redistribution during the glacial process the initial sea level has to be determined iteratively (e.g., Kendall et al., 2005).

To circumvent this problem, we consider the RSL rate, i.e. its time derivative, which depends much less on the initial state. Although knowing that this quantity has to be derived from a series of SLIPs resulting in increased errors, we consider it as a tractable procedure. Here, the SLIP linear rate at $t_{n}$ is determined from the RSL difference at $t_{n}$ and $t_{n-1}$. The RSL data used to compute the rates for the assimilation (which function as our observations) are taken from the reference run, $m_{0}$ (cf. Fig. 3). This synthetic data set is therefore well known and its statistical properties can be linked to the assimilation results.

The spatial distribution of collected SLIPs is rather heterogeneous, mainly spreading along the coasts of the continents, at islands, and concentrating to regions of large ice-water changes since the LGM. In order to run realistic scenarios, the synthetic observations were limited to locations where such data are available in reality. The dynamic model is discretized on a global $256 \times 512$ grid. The grid point closest to each SLIP site was chosen for the representation of the synthetic data. That way, 1807 observation points were obtained. They are shown in Fig. 4. At each observation point we constructed a time series from the complete synthetic record, i.e. without gaps.

\subsection{Investigated setups}

This study consists of three setups. Setup 1 investigates the influence of observation uncertainty on the assimilation. In the second setup the observations were restricted to certain regions in order to test the performance of our approach when observations are not available globally. In the third setup, the time interval with available observations was restricted to after $10 \mathrm{ka}$ BP until present day.

The first setup is split into two scenarios based on the Cases A-D listed in Table 2. The purpose is to investigate the influence of observation uncertainty and statistical parameters of the initial ensemble on the convergence and uncertainty of the viscosity estimates. 


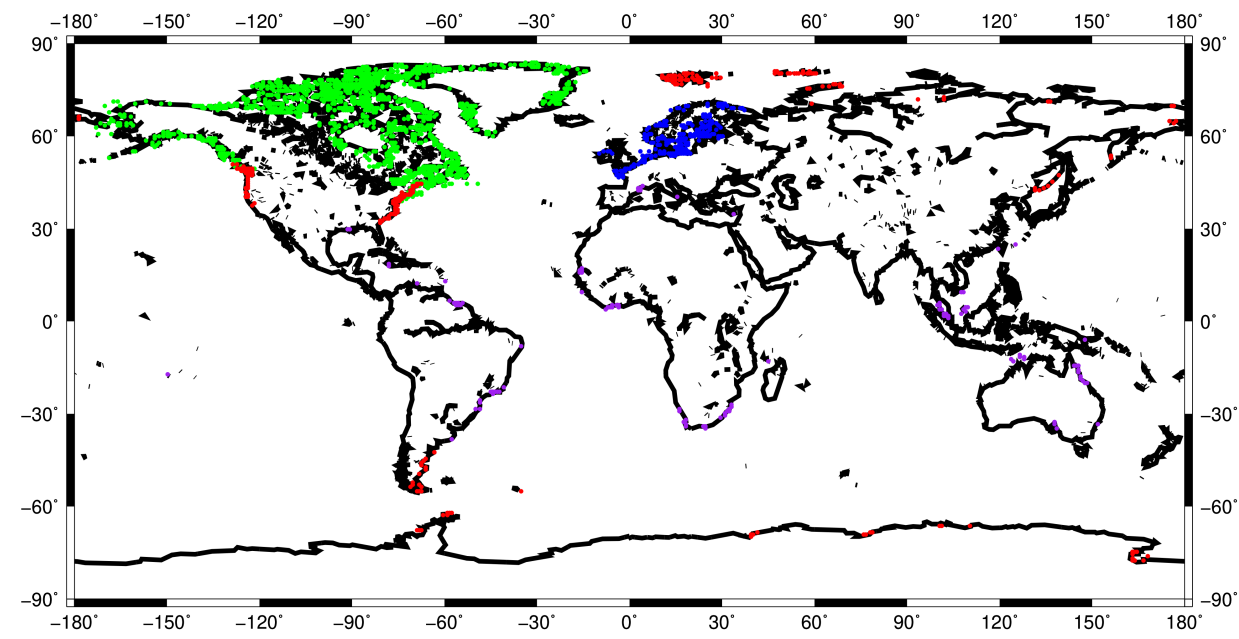

Figure 4. Locations of synthetic observations. The chosen points are locations of existing sea level index points. They are unevenly distributed and located mostly along the coasts of regions where large sea level changes have occurred in the past or are still ongoing. The observation locations where sub-divided into four regions: Laurentide \& Greenland (green), Fennoscandia (blue), Far-field (purple), and other (red).

In scenario one (Cases A-C), the initialization is equal in all three cases: perturbation with noise drawn from a normal distribution $N\left(\mu_{\text {init }}, \sigma_{\text {init }}\right)$ such that $\left|\nu_{\text {target }}-\mu_{\text {init }}\right|=\sigma_{\text {init }}$. The Cases A-C differ only in the observation uncertainty. It is varied from $0.1 \mathrm{~m}$ in case A to $0.5 \mathrm{~m}$ in Case $\mathrm{C}$. If $\mu_{\text {init }} \leq \sigma_{\text {init }}$ the target viscosity value is well covered by the ensemble PDF and the influence of different observation uncertainties can be studied.

In scenario two (Cases B and D), the influence of the initial offset is studied. We compare test Case B (with a moderate initial offset) to a case where a large offset $\left(\mu_{\text {init }}=2 \sigma_{\text {init }}\right)$ was chosen, such that the target viscosity value lies in a tail of the ensemble PDF. Having in mind a future application of the method to real data it is important to also reach convergence if the true value lies somewhere in a tail of the initial ensemble PDF. One must ensure that the true value is still properly sampled by the ensemble PDF. Otherwise the filter degenerates. Therefore, the true value must not lie outside the ensemble. But when the target value is unknown, it cannot be ensured that it lies somewhere near the ensemble mean but is is also possible that it is located somewhere in a tail of the chosen initial PDF.

For the assimilation in setup 2, four sets of observations where compiled. In the first set, all available observations where used. This gives the best possible global coverage with observations. In three following scenarios, observations where restricted to 1) Laurentide and Greenland (Case LG), 2) Fennoscandia and Northern Europe (Case FS), or 3) the far-field. This was done to investigate under which conditions in the 1D model setup regional observations can be used to obtain correct global viscosity values. When considering real SLIPs, observations might be available only in certain parts of the world and it is important that our approach is proven successful under those conditions.

Looking at the temporal distribution of real SLIP observations it becomes clear that most of them date from after the last glaciation. This is due to the fact that during the glaciation period the ice cover prevented the formation of SLIPs at many of the 
most interesting locations. Therefore, in setup 3 we tested our approach for the case of observations being available only after $10 \mathrm{ka} \mathrm{BP}$. The parameters of the test in those cases correspond to Cases B10 and C10 with observation uncertainties of $0.25 \mathrm{~m}$ and $0.5 \mathrm{~m}$, respectively (see Table 2). In this time period, RSL is mainly dominated by the Earth's deformation (post-glacial rebound) and less by changes in barystatic sea level.

In general, large ensemble sizes (especially for high-dimensional problems) are necessary to properly sample the model PDF. Due to the low dimensionality of the problem described here (only two distinct viscosity values) an ensemble size of 50 proved to be sufficient in the presented experiments.

\section{Results}

\subsection{Consistency tests (Setup 1)}

In setup 1 , we studied the convergence of the weighted mean to the target values of the reference model when all available observations from the time interval $25.5 \mathrm{ka} \mathrm{BP}$ until present day are considered. Figure 5 shows the misfit measure development over time for scenario one (Cases A-C, cf. Table 2). The root-mean-square (RMS) of the difference between sea level observations and model predictions of each ensemble member drops quickly after the onset of the assimilation and the first resampling. The very improbable particles from the initial ensemble are disregarded already at this step. In the course of the following assimilation the RMS stays mostly below $0.5 \mathrm{~m}, 1 \mathrm{~m}$, and $1.5 \mathrm{~m}$ for Cases A, B, and C, respectively, and converges to values of $0.25 \mathrm{~m}, 0.6 \mathrm{~m}$, and $0.9 \mathrm{~m}$ towards the end of the assimilation period. In all cases, there is a prominent RMS error peak at about $13.5 \mathrm{ka} \mathrm{BP}$ and a minor peak around $10 \mathrm{ka} \mathrm{BP}$.

Figure 6 shows the corresponding development of the viscosity values. In all Cases $A-C$ we observe very good convergence to the the upper and lower mantle viscosities of the reference model. From a state with a large variance the ensembles evolve to a state with much lower variance that is governed by the observation uncertainties and model errors. The ensemble means converge towards the target values and stabilize within a range below $\pm 5 \%$ difference to the reference value for the lower mantle and $\pm 2 \%$ for the upper mantle. In cases B and C the ensemble spread is larger than in case A. Nevertheless, the ensemble mean is able to recover the target viscosities of the reference run within some error margin. The recovered mean values (weighted ensemble means) also lie within $\pm 5 \%$ and $\pm 1 \%$ of the target values of lower and upper mantle viscosities, respectively. The viscosity values of the upper mantle region converge more quickly than those of the lower mantle.

In scenario two (Cases B and D) we verify ensemble convergence for the case of the target viscosity value being in the tail of the initial ensemble PDF. That means we added an offset to the initial viscosities such that the target viscosities are far from the initial ensemble mean. Figure 7 shows the RMS error of the model predictions with respect to the observations for the two cases compared in this scenario. Both ensembles converge to model states that yield RMS values of about $0.6 \mathrm{~m}$. The RMS development of Case D is similar to Case B, only the initial errors are higher in Case D. The parameters of Cases B and D differ only in the initial mean value of the ensemble perturbation $\mu_{\text {init }}$ (cf. Table 2).

215 Figure 8 shows the development of the viscosities in the ensemble for the test cases of scenario two. The ensemble mean of Case D converges equally well as the one of Case B. Also, the ensemble spread is of the same order for both test cases. 
https://doi.org/10.5194/npg-2021-22

Preprint. Discussion started: 7 June 2021

(c) Author(s) 2021. CC BY 4.0 License.

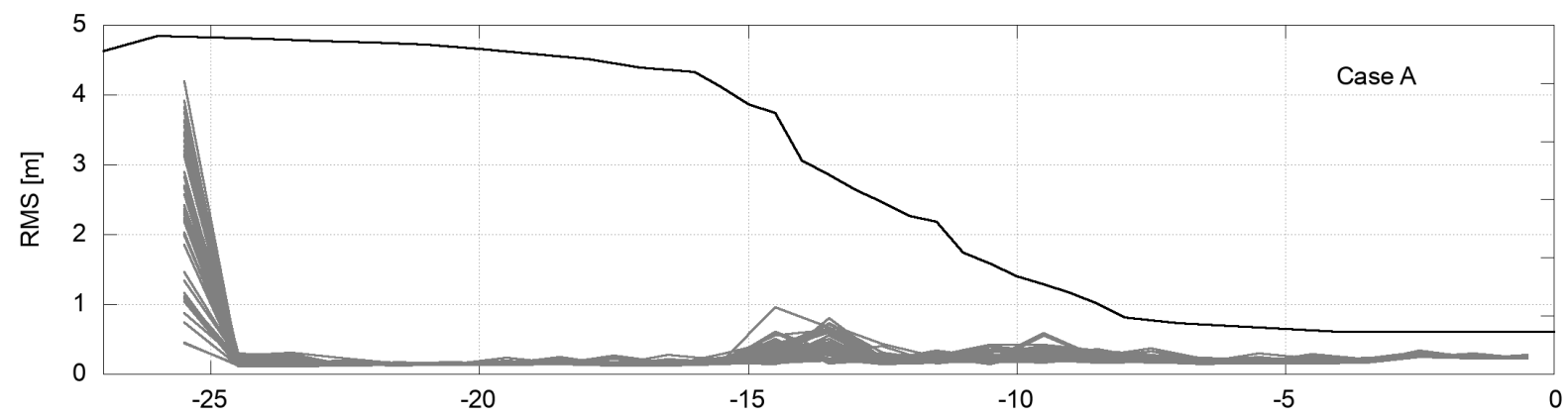

$8 e+07$

$7 e+07$

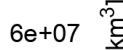

$5 e+07$

$4 e+07$

$3 e+07$

$2 e+07$

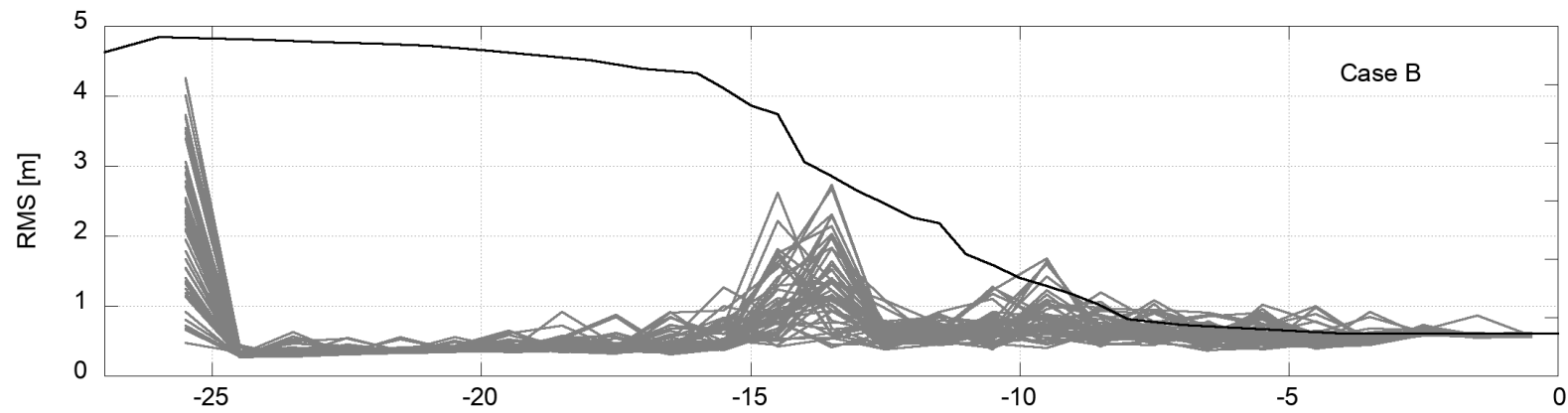

$8 e+07$

$7 e+07$

$6 e+07$ है

$5 e+07$

$4 \mathrm{e}+07$

$3 e+07$

$2 e+07$

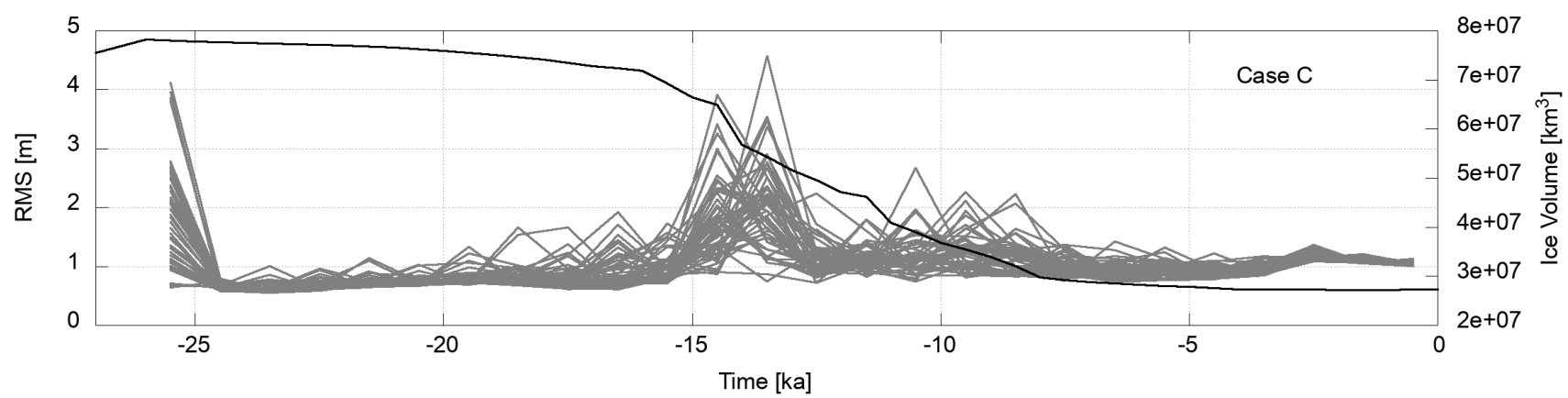

Figure 5. Measures of misfit development for the ensemble of Setup 1 (cases A-C) in gray. Shown are the RMS values of the difference between the sea level observations and the model predictions of each ensemble member. The black lines show the total ice volume according to the external ice model. There are spikes following large changes in total ice mass. 

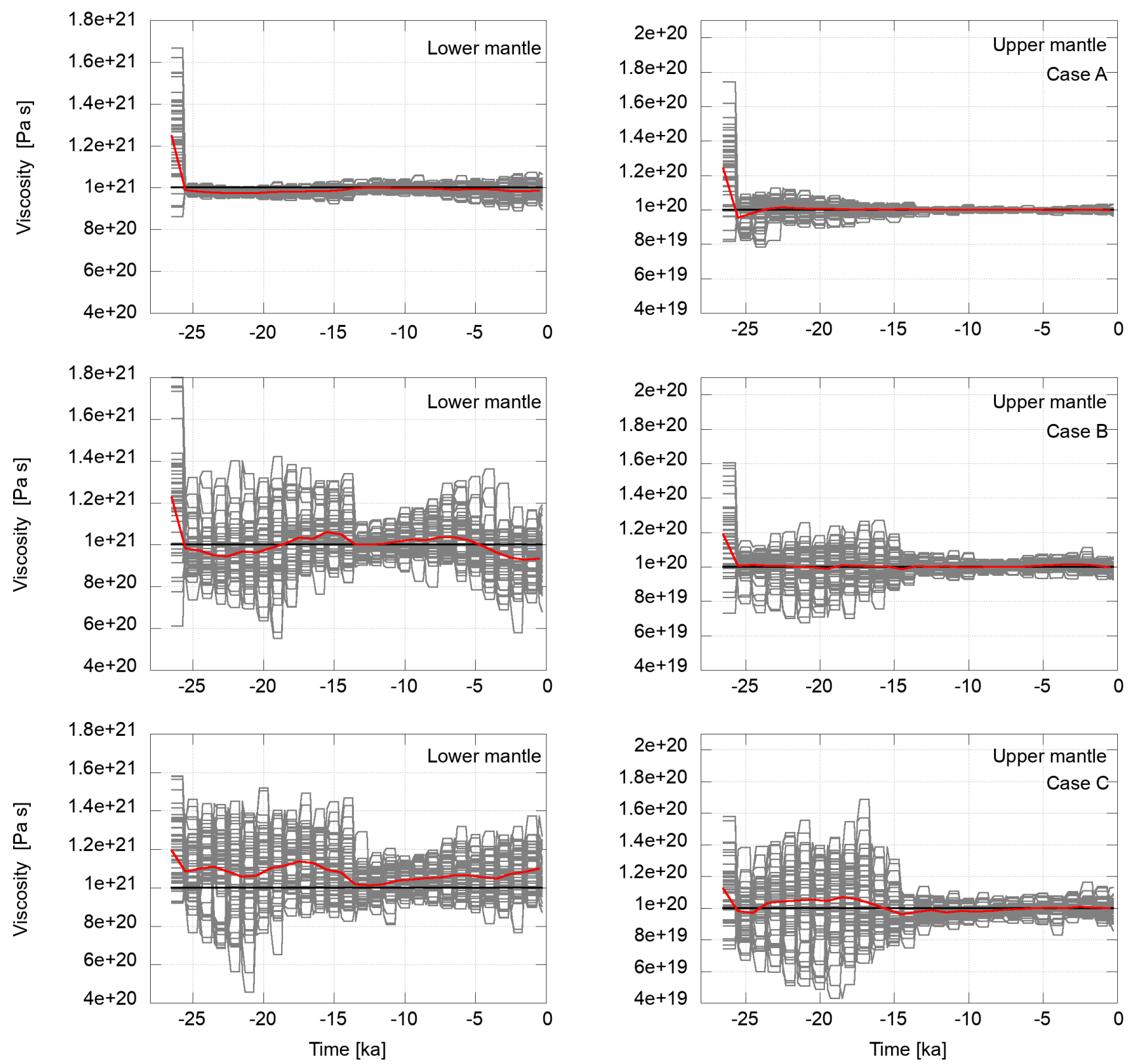

Figure 6. Development of viscosity in the lower mantle (left column) and the upper mantle (right column) for scenario one (Cases A-C, from top to bottom) in gray. The flat segments represent the viscosity values of an ensemble member during the forecast phase. When observations are available, a model state may be resampled and perturbed. This changes the viscosity values as shown. The horizontal black lines represent the viscosities of the reference experiment towards which the ensemble mean is expected to converge. The red lines are the weighted ensemble means. Ensemble members are weighted by the likelihood of the observations given the current member model state. 


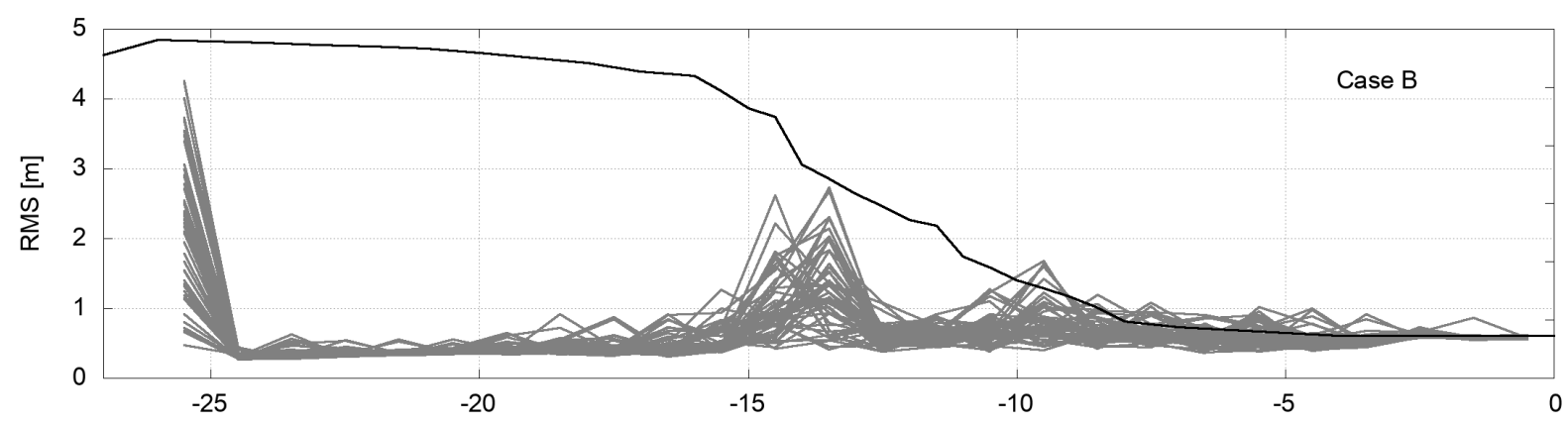

$8 \mathrm{e}+07$

$7 e+07$

$6 e+07$

$5 e+07$

$4 \mathrm{e}+07$

$3 e+07$

$2 \mathrm{e}+07$

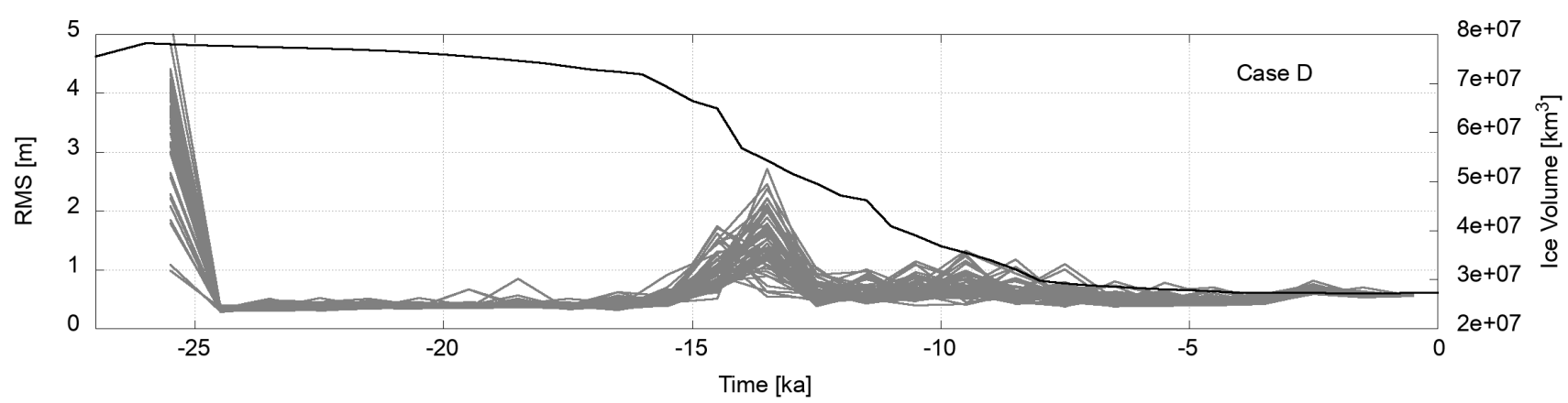

Figure 7. Measures of misfit development for the ensemble of scenario two in Setup 1 (Cases B and D) in gray. Shown are the RMS values of the difference between the observations and the model predictions. The black lines show the total ice volume according to the external ice model.

Therefore, if the initial ensemble's sampling density in the neighborhood of the target value is high enough, the filter does not degenerate and the subsequent behavior is similar to the test case with lower initial offset. In that case the weighted ensemble mean converges to the target value.

The variance or STD of the ensemble represents the uncertainty of the parameter estimation. Figure 9 shows the STD development for the ensemble in each test case of Setup 1 . The two mantle regions are shown separately since their viscosity magnitudes are very different. In both regions there are two time ranges with quite different STD levels. The first region with higher STD levels lasts until about 14.5 ka BP. After that, STD levels drop in all test cases and rise slightly towards the end of the assimilation period.

\subsection{Regional observations (Setup 2)}

In the first regional test (Case LG) we restricted the observations to those located in the area of the Laurentide ice sheet and Greenland (see Fig. 4). There are 1309 locations with observations in that region in our data set. In Case FE only 209 locations from Fennoscandia and Northern Europe are considered. The statistical parameters for the regional cases are equal to those of Case B (cf. Table 2). 

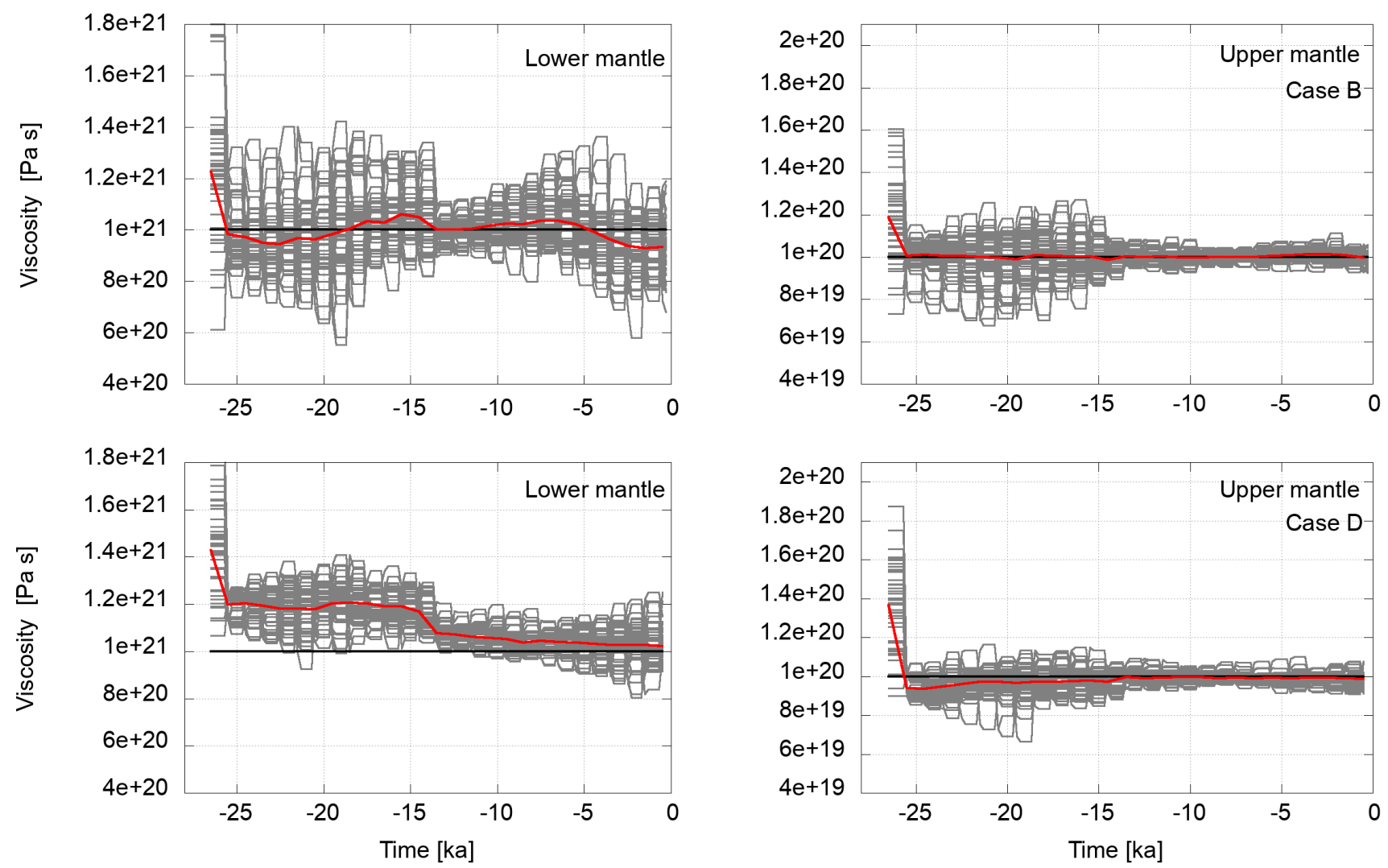

Figure 8. Development of viscosity in the lower mantle (left column) and the upper mantle (right column) for scenario two (test cases B and $\mathrm{D}$, from top to bottom) in gray. The flat segments represent the viscosity values of an ensemble member during the forecast phase. When observations are available, a model state may be resampled and perturbed. This changes the viscosity values as shown. The horizontal black lines represent the viscosities of the reference experiment towards which the ensemble mean is expected to converge. The red lines are the weighted ensemble means. For the initial value all members are weighted equally. Thereafter, they are weighted by the likelihood of the observations given the current member model state. 

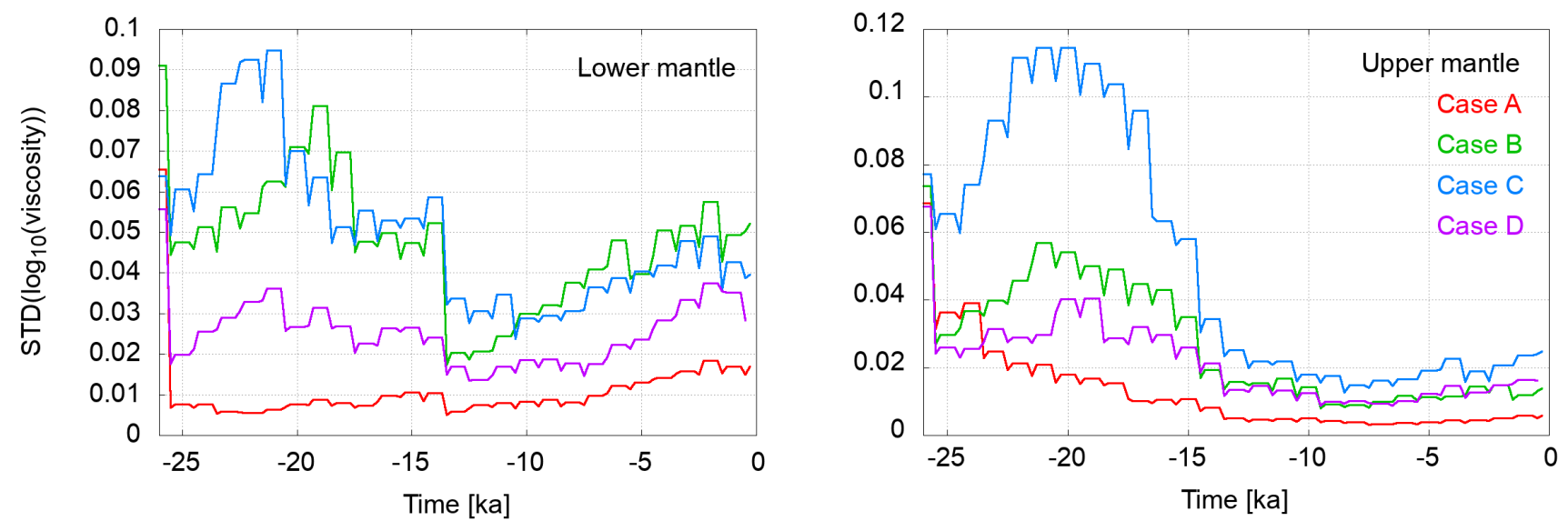

Figure 9. Development of the ensemble standard deviation of $\log _{10}(\nu)$ over time (red: Case A, green: Case B, blue: Case C, purple: Case D). The left panel shows the values for the lower mantle and right panel for the upper mantle, respectively. The points at the beginning and end of each plateau represent the ensemble at the beginning and end of a forecast phase. They are equal since during the forecast the viscosity remains unchanged. The drop after a plateau happens due to resampling and the subsequent rise due to perturbation (although this happens at the same point in time, the values are shifted horizontally to visualize the development).

Figure 10 shows the development of the RMS error for the RSL observations for the Cases LG and FS. The RMS development is similar to the development in Setup 1, where the full data set is applied (see top panel of Fig. 7). In case of the complete data set, the final RMS values at present day are about $0.6 \mathrm{~m}$. This also holds for Case LG. The Fennoscandian data set with considerable fewer observations distributed over a smaller area shows slightly larger RMS values for the peaks at $15 \mathrm{ka}$ and $9 \mathrm{ka} \mathrm{BP}$ and also at present day (about $0.9 \mathrm{~m}$ ).

Figure 11 shows the model state development of the viscosity values of each ensemble member and the weighted mean. Clearly, the weighted means converge towards the target values. The convergence of lower mantle viscosity is slower and shows more variability than the upper mantle viscosities. For the North America and Greenland region, the development over time shows the same characteristics as in the complete data set experiments. There is a period with large variability until about 13.5 ka BP after which it drops significantly. In case of Fennoscandia, there is no such drop-off in variance (see also Fig. 12) in the lower mantle viscosities. It is present, though less prominent, in the upper mantle viscosities.

\subsection{Time interval tests (Setup 3)}

In Setup 3 only observations taken after $10 \mathrm{ka}$ BP where used in the assimilation. This corresponds to times after the last deglaciation. With this setup we demonstrate that the algorithm can reach convergence in a short period of time that is very relevant for real observations. 


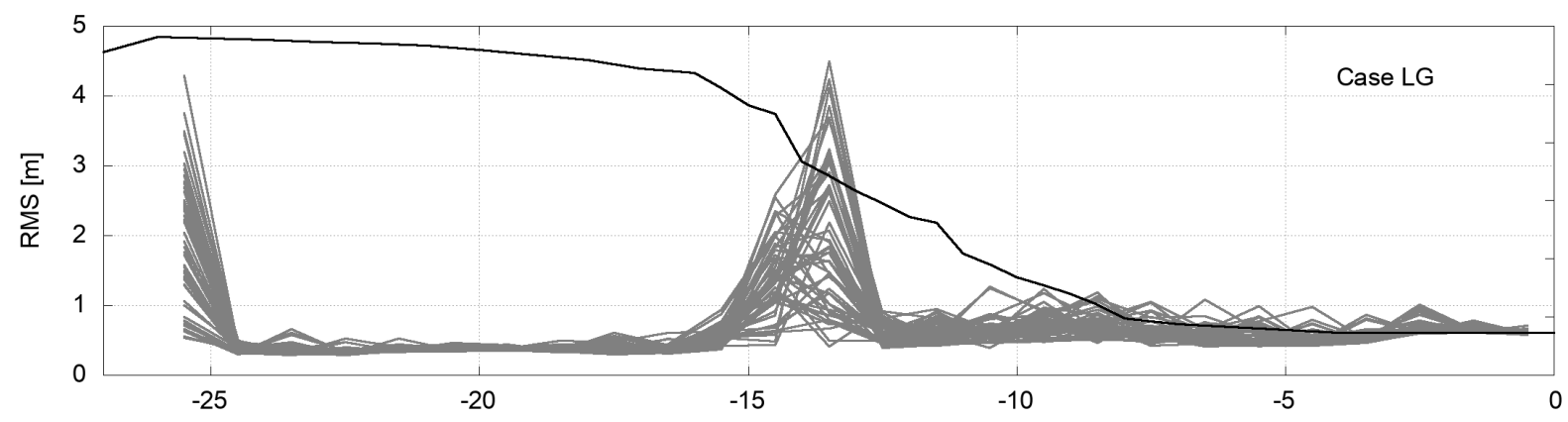

$8 \mathrm{e}+07$

$7 e+07$

$6 e+07$

$5 e+07$

$4 \mathrm{e}+07$

$3 e+07$

$2 \mathrm{e}+07$

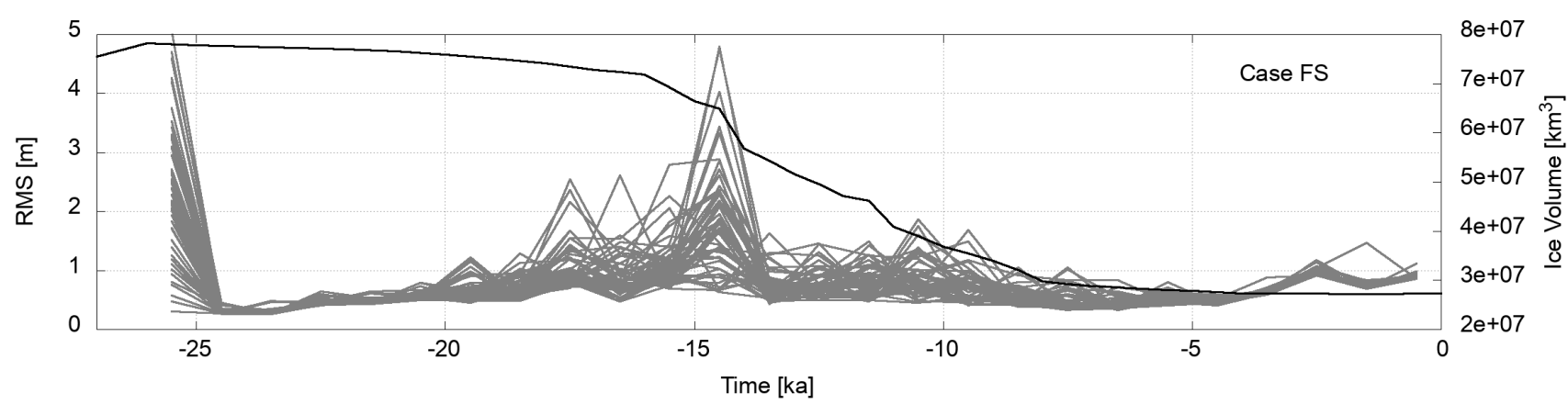

Figure 10. Measures of misfit development for the ensemble of two regional observation sets: Northern America and Greenland (left) and Fennoscandia (right). Shown are the RMS values of the difference between the observations and the model predictions. The black lines show the total ice volume according to the external ice model. There are spikes following large changes in total ice mass that can be explained by different response times of the reference model and the ensemble member to changing mass load. The response time depends on the mantle viscosity of the individual model.

Figure 13 shows the development of the RMS misfit of RSL for Setup 3. The final RMS values are in the same range $(0.5 \mathrm{~m}$ for Case B10 and $1 \mathrm{~m}$ for Case C10, respectively) as the values for the corresponding cases considering observations from $25.5 \mathrm{ka} \mathrm{BP}$ to present day. There is little variability within the ensembles. There are no RMS spikes in this time period.

Figure 14 shows the development of viscosity values over time. The convergence of the weighted mean to the target values is very fast. Although there is some variability within the ensemble the weighted mean is very stable over time.

Figure 15 shows the development of the ensemble's viscosity STD over time. There is a quick drop-off after assimilation onset. Thereafter, the STD stays fairly constant until the end of the assimilation. Only in Case C10 a slight rise of STD is visible in the upper mantle. We see the same features in the STD in Setup 1 where the entire data set was used (see Fig. 9 but the variability is slightly lower in this $10 \mathrm{kyr}$ case. 

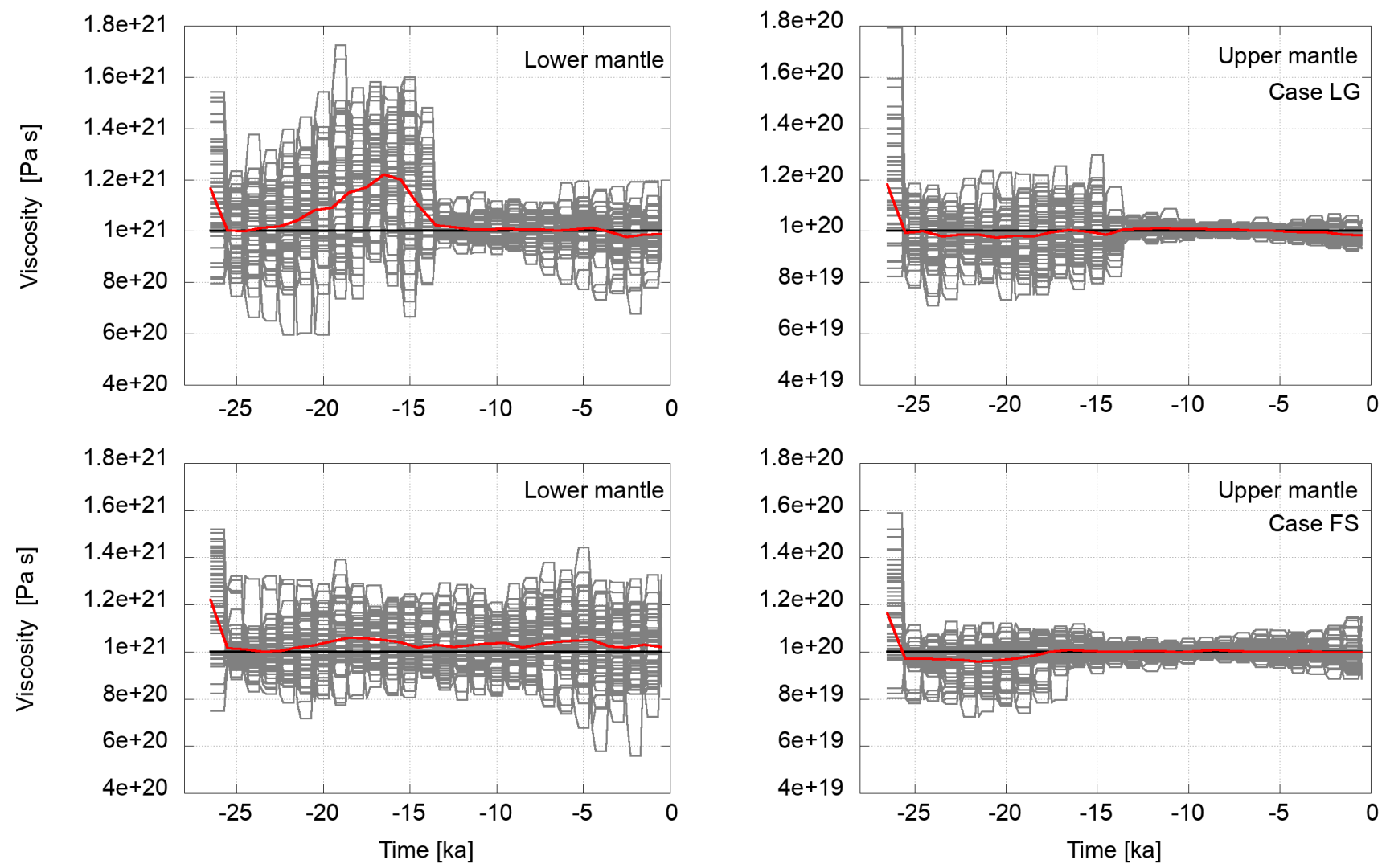

Figure 11. Development of viscosity in the lower mantle (left column) and the upper mantle (right column) for test cases with regional data sets: Northern America and Greenland (top row) and Fennoscandia (bottom row). The flat segments represent the viscosity values of an ensemble member during the forecast phase. When observations are available, a model state may be resampled and perturbed. This changes the viscosity values as shown. The horizontal black lines represent the viscosities of the reference experiment towards which the ensemble mean is expected to converge. The red lines are the weighted ensemble means. For the initial value all members are weighted equally. Thereafter, they are weighted by the likelihood of the observations given the current member model state. 

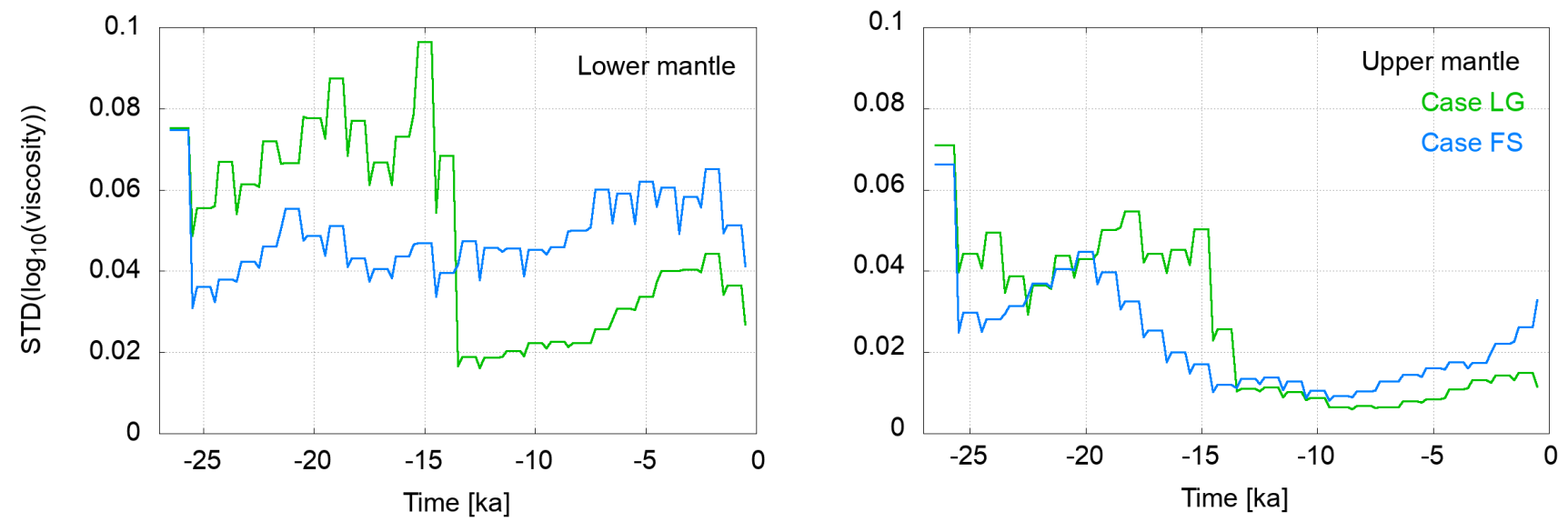

Figure 12. Development of the ensemble standard deviation of $\log _{10}(\nu)$ over time (green: Laurentide \& Greenland, blue: Fennoscandia). The left panel show the values for the lower mantle and right panel for the upper mantle, respectively. The points at the beginning and end of each plateau represent the ensemble at the beginning and end of a forecast phase. They are equal since during the forecast the viscosity remains unchanged. The drop after a plateau happens due to resampling and the subsequent rise due to perturbation (although this happens at the same point in time, the values are shifted horizontally to visualize the development).

\section{Discussion}

The results of setup 1 show that the weighted ensemble mean converges to the target values. The final misfit of RSL and the ensemble variance scales with the assumed observation uncertainty. This is expected since with increasing observation uncertainty the correction of the dynamic models in the assimilation step is reduced. In the particle filter we used, ensemble members with low likelihood are resampled to model states with high likelihood. Larger observation uncertainties reduce the separability of models based on that measure. As a consequence less models are resampled to better model states, the convergence slows down and the final ensemble shows a larger variability.

Although in general the convergence of the ensemble is very good, there are some RMS peaks at about $13.5 \mathrm{ka} \mathrm{BP}$ and $10 \mathrm{ka}$ BP that appear suddenly and slow down the convergence. These peaks coincide with larger changes in ice volume (melt water pulses) with a delay of 1 to 2 kyrs. They can be explained by the different response times to the load change, which depend on the mantle viscosity of the individual model. The general level of misfit, as well as the peaks after sudden changes in ice volume, scale with the observation uncertainty assumed in that specific test case. This result shows, the interplay between melt water and the Earth's response hinders the inference of structural parameters during this phase as the barystatic sea level change dominates, which is independent of the Earth's structure.

The convergence of the viscosities to the target values is very good. Generally, the convergence decreases slightly from Case A to C. This is due to the larger observation uncertainties which allow particles to survive which are farther from the target model. The viscosities in the lower mantle show slower convergence than in the upper mantle. The reason for that is 

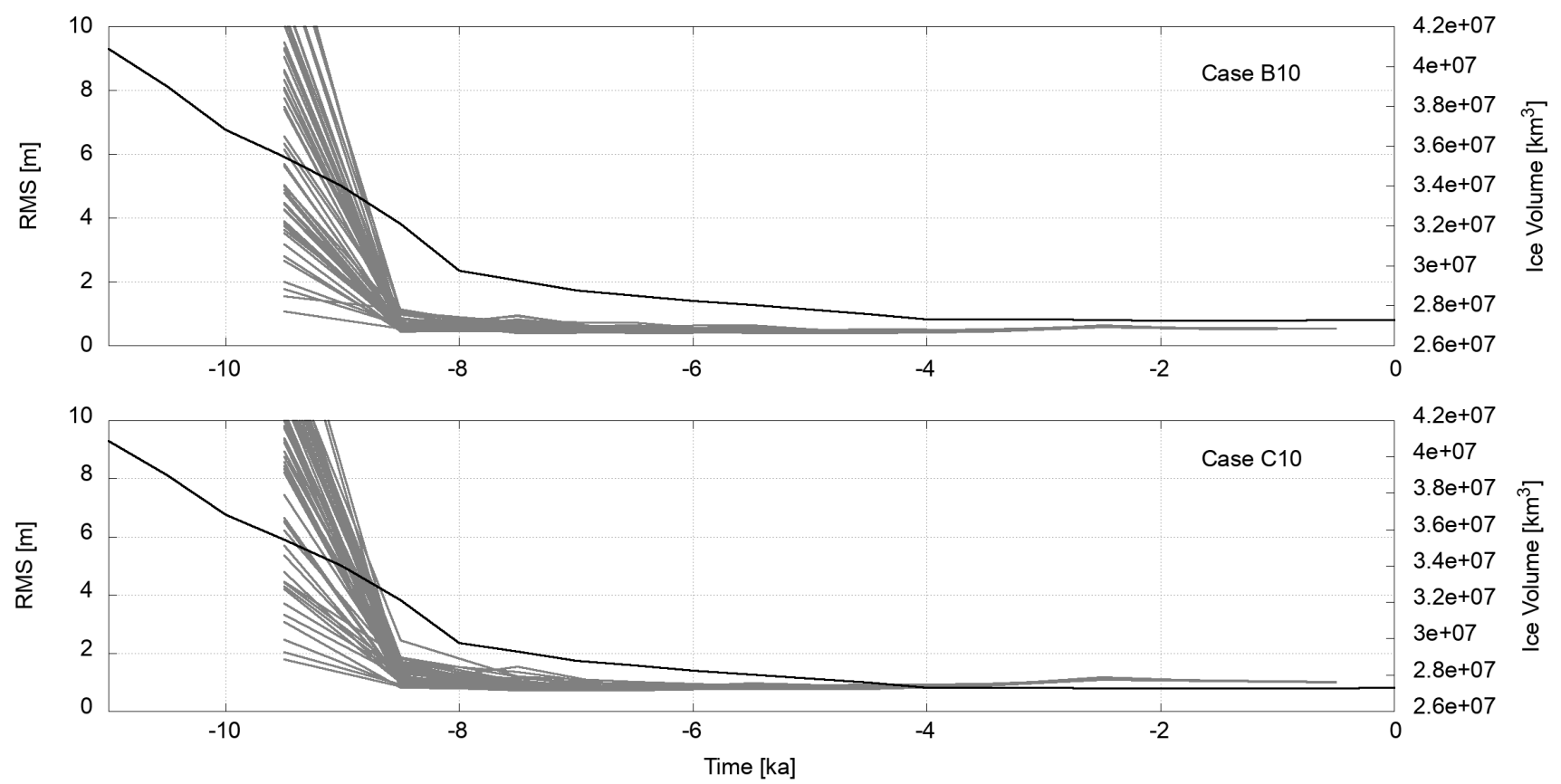

Figure 13. Measures of misfit development for the ensemble of two sets of observations dating from after $10 \mathrm{ka}$ BP. The statistical parameters of Cases B10 and C10 are equal to those of Cases B and C in Table 2. The assumed observation uncertainties are $0.25 \mathrm{~m}$ (top) and $0.5 \mathrm{~m}$ (bottom) Shown are the RMS values of the difference between the observations and the model predictions. The black lines show the total ice volume according to the external ice model.

that viscosity changes in the lower mantle take more time to have impact on sea levels. In general, small deformations at the surface have little impact on lower mantle deformation and with increasing depth it becomes more difficult to constrain mantle viscosity by surface deformation. This is also apparent when looking at the variability within the ensemble as shown in Fig. 9. The slightly rising variability towards the end of the assimilation period might be due to the low magnitude of RSL change rates in younger times. With very small signals it is difficult to correct the models properly and the variance introduced by the perturbation leads to a slight rise in variance within the ensemble.

The convergence of the ensemble mean viscosities to the target values of the reference runs in the presented cases show that with our approach we are able to recover mantle viscosities within a reasonable uncertainty range. This is even the case if the initial ensemble's PDF is far from the target values, i.e. the target value is in one of the tails of the initial PDF. A requirement is, however, that the sampling density of the ensemble near the target value is still high enough such that the filter does not degenerate. Furthermore, the convergence is strongly influenced by the assumed observation uncertainties. Large uncertainties on the one hand slow down the convergence and lead to larger final variance within the ensemble. On the other hand they reduce the chance of degeneration since particles with larger deviations from the target values are assigned higher likelihoods if observation uncertainties are higher. 

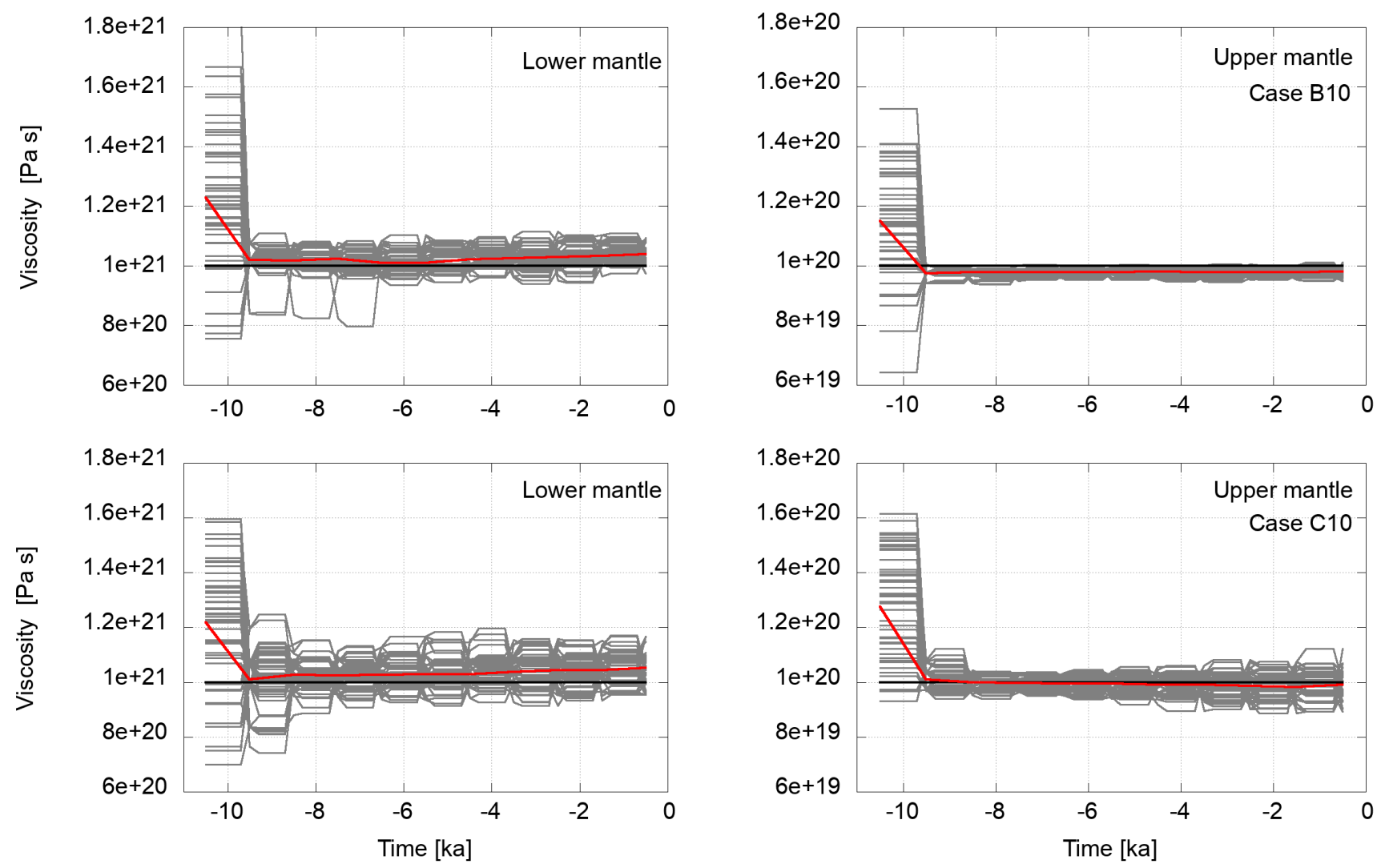

Figure 14. Development of viscosity in the lower mantle (left column) and the upper mantle (right column) for test cases with observations starting from $10 \mathrm{ka} \mathrm{BP}$. Observation uncertainties are $0.25 \mathrm{~m}$ (left column) and $0.5 \mathrm{~m}$ (right column). The flat segments represent the viscosity values of an ensemble member during the forecast phase. When observations are available, a model state may be resampled and perturbed. This changes the viscosity values as shown. The horizontal black lines represent the viscosities of the reference experiment towards which the ensemble mean is expected to converge. The red lines are the weighted ensemble means. For the initial value all members are weighted equally. Thereafter, they are weighted by the likelihood of the observations given the current member model state. 

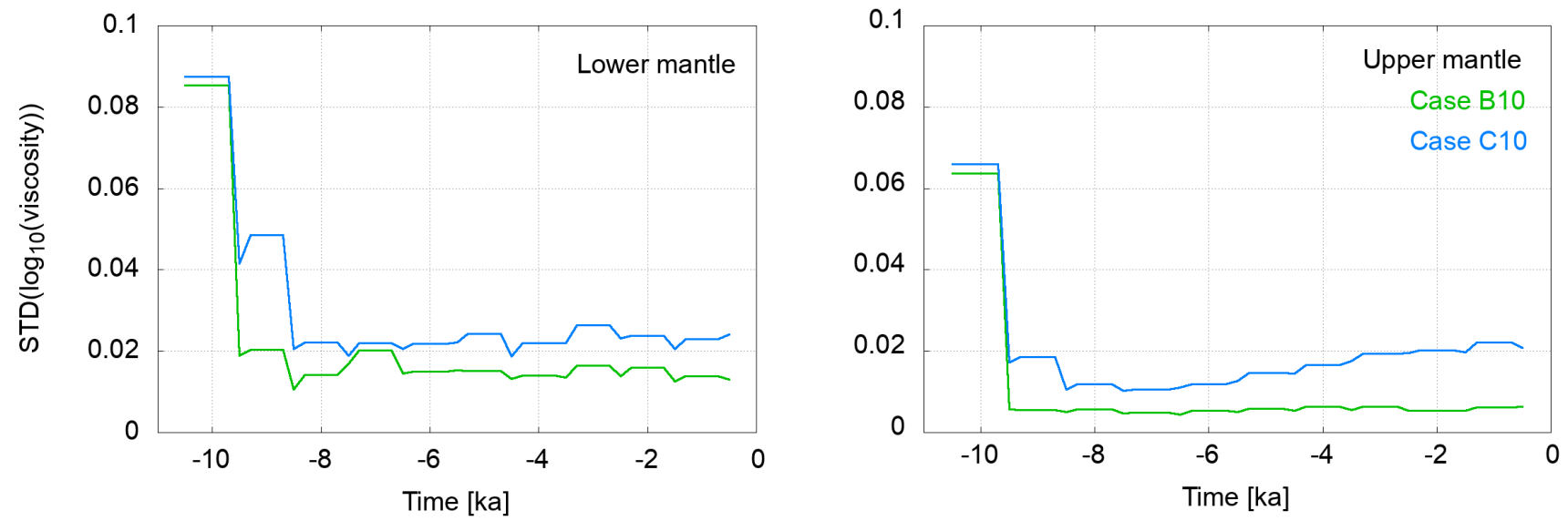

Figure 15. Development of the ensemble standard deviation of $\log _{10}(\nu)$ over time for the $10 \mathrm{kyr}$ assimilation (green: Case B10, blue: Case C10). The left panel shows the values for the lower mantle and right panel for the upper mantle, respectively. The points at the beginning and end of each plateau represent the ensemble at the beginning and end of a forecast phase. They are equal since during the forecast the viscosity remains unchanged. The drop after a plateau happens due to resampling and the subsequent rise due to perturbation (although this happens at the same point in time, the values are shifted horizontally to visualize the development). uncertainty of the final estimations seems to depend only little on the size of the observation region. The 3-layer model with two mantle layers is simple enough to recover the viscosities with only little more than 200 observation locations. However, there are differences in the lower mantle viscosity estimation. The larger variance in lower mantle viscosity from Fennoscandia when compared to Laurentide can be explained by the smaller size of the Fennoscandian ice sheet. Accordingly the GIA of Fennoscandia is less sensitive to lower mantle viscosity structure (e.g. Mitrovica and Peltier, 1993; Lambeck et al., 1998). For the upper mantle there seems to be only little difference in the viscosity variance between the two cases. For both regions, signals from their respective ice shields are large enough to constrain the Earth structure.

In setup 3 we show that it is possible to estimate the target viscosities also when only observations from a short time interval, i.e., from $10 \mathrm{ka}$ BP until present day are available. The reasoning behind those test cases is that the $10 \mathrm{ka}$ BP marks the end of the last deglaciation and most real observations date from after that. The RMS misfit of RSL obtained in these tests drop quickly after the onset of the assimilation. There are no large ice mass changes after $10 \mathrm{ka}$ BP. The only ongoing process is the post-glacial rebound. All model realizations were able to reproduce that (relatively slow) GIA processes taking place and there is little variance in the ensemble. Such behavior was already observed in Setups 1 and 2 where the variance also drops significantly after $10 \mathrm{ka} \mathrm{BP}$.

The results of this experiment show that our algorithm can quickly converge to the target values of the reference model under quiet conditions. With "quiet" we mean there are no large changes in global ice mass within a short period of time and therefore the models have enough time to adapt to the new mass load. In that case, the RSL development is strictly a function of 
viscosity describing an exponential decrease (the relaxation process). On the other hand, if larger ice mass changes are present, the algorithm also converges but it takes longer and several assimilation steps are needed until the models adapt to the new mass load.

\section{Conclusions}

We have shown that our algorithm is able to recover mantle viscosities through assimilation of paleo sea level rates of change. This is the case even if the target viscosities are located in a tail of or even outside the initial distribution. This way, in contrast to the common approach, the final viscosity values need not be included in the initial guess, which has the potential to lead to viscosity values that are closer to the truth than any member of the initial ensemble.

However, some of the assumptions made about observation uncertainties need closer inspection. Real SLIP uncertainties are usually in the range of $0.5 \mathrm{~m}$ to $1 \mathrm{~m}$ for stratigraphic data and as precise as $0.1 \mathrm{~m}$ to $0.2 \mathrm{~m}$ for a few very exact data points, only (depending on the stratigraphic regime and the age of the SLIP) (Shennan et al., 2015, pp. 3-25). We considered uncertainties of $0.25 \mathrm{~m}$ to $0.5 \mathrm{~m}$ for all observations. This seemed to be a good compromise given the range of real SLIP uncertainties. However, in addition to SLIPs, which are defined as band limits of paleo sea level, other sea level data only indicate an upper or lower bound of paleo sea level (e.g. Khan et al., 2015). Accordingly, we have to extend the error handling towards non-Gaussian error distributions (e.g. Hibbert et al., 2016; Latinović, 2021).

The ensemble size was limited to 50 members, mainly due to computation time and memory usage. For a proof of concept with synthetic data this ensemble size is sufficient. In order to recover real viscosities and as target values are unknown, a larger ensemble sampling a wider range of viscosity values will be necessary.

The focus of GIA related sea level research lies on reconstructions of the deglaciation since the last glacial maximum (e.g., van de Plassche, 1986; Düsterhus et al., 2016; Carlson et al., 2019), which constrains the main range of available sea level data to be younger than $20 \mathrm{ka}$ BP. While we considered a spatial distribution based on available data sets (cf. Unger et al., 2012)), we assumed to have observations every $1 \mathrm{kyr}$ for the whole assimilation period. The amount of available sea level data, however, peaks during late Holocene at $\sim 8$ to $12 \mathrm{ka} \mathrm{BP}$ and is considerable smaller for earlier periods.

Another crucial point is that we compute rates of sea level change from two observations at a given location. The linear rate is attributed to the younger boundary of the time interval while it really is a mean value for the whole interval. This introduces errors in time of observation and magnitude of sea level change. This is not a problem in our twin-experiment since observations and model predictions are treated the same way. However, if real SLIP data are used, there are additional dating errors for the sea level estimates. They have an impact on the rate of change uncertainty and their consequential influence on the uncertainty of the viscosity estimates is a point future investigations need to pay attention to.

In this first approach we cover a rather simple 1D Earth structure with two mantle layers of constant viscosity and did not consider uncertainty in lithosphere thickness. While this is helpful for the algorithm development, more realistic scenarios involve radial viscosity profiles and even 3D viscosity variations. For a profound impact on viscosity parameter estimation and regional sea level changes this is an issue that will be addressed in future work. 
https://doi.org/10.5194/npg-2021-22

Preprint. Discussion started: 7 June 2021

(c) Author(s) 2021. CC BY 4.0 License.

(c) (i)

Available SLIPs are sparse in the distant past and become more numerous as recent time is approached. But the situation improves due to various groups working on constraining paleo sea level rise under PAGES like HOLSEA and PALSEA (Carlson et al., 2019).

Nowadays, even more sea level and ice-mass data based on GPS, tide gauges or measurements of mass redistribution with satellite missions such as GRACE and GRACE-FO are available. The incorporation of such data in the assimilation will reduce the uncertainty of the estimated parameters further.

Data availability. The synthetic data set used in this study has been submitted to a data repository at Helmholtz Centre Potsdam GFZ. The DOI will be made available when the paper is accepted.

Author contributions. RS has been responsible for the methodology, investigation, formal analysis, visualization, and preparation of the initial manuscript draft. JS was responsible for conceptualization, methodology, project administration, and project supervision.VK has contributed the code for the VILMA model and supported the methodology. MB has provided auxiliary software, and supported the investigation.MT has been involved in project administration and funding acquisition. All authors have been involved in reviewing and editing the manuscript.

Competing interests. The authors declare that they have no conflict of interests.

Acknowledgements. The work described in this paper has received funding from the Initiative and Networking Fund of the Helmholtz Association through the project "Advanced Earth System Modelling Capacity (ESM)". The numerical simulations were performed at the German Climate Computing Center (DKRZ). Parts of the study are based on work funded by the German climate modeling project PalMod (FKZ: 01LP1502E, 01LP1503A) and PalMod II (FKZ: 01LP1918A) that was supported by the German Federal Ministry of Education and Research (BMBF) as a Research for Sustainability initiative (FONA). Some figures were produced using the Generic Mapping Tool (GMT) by Wessel et al. (2019). 
https://doi.org/10.5194/npg-2021-22

Preprint. Discussion started: 7 June 2021

(c) Author(s) 2021. CC BY 4.0 License.

\section{References}

Anderson, B. D. and Moore, J. B.: Optimal filtering, Prentice-Hall, Englewood Cliffs, NJ, 1979.

Asch, M., Bocquet, M., and Nodet, M.: Data assimilation: methods, algorithms, and applications, SIAM, 2016.

Bärenzung, J., Holschneider, M., Wicht, J., Sanchez, S., and Lesur, V.: Modeling and Predicting the Short-Term Evolution of the Geomagnetic

Field, J. Geophys. Res-Sol. Ea., 123, 4539-4560, https://doi.org/10.1029/2017JB015115, 2018.

Bauer, P., Thorpe, A., and Brunet, G.: The quiet revolution of numerical weather prediction, Nature, 525, 47-55, https://doi.org/10.1038/nature14956, 2015.

Box, G. E. P. and Tiao, G. C.: Bayesian inference in statistical analysis, vol. 40, John Wiley \& Sons, 2011.

Carlson, A. E., Dutton, A., Long, A. J., and Milne, G. A.: PALeo constraints on SEA level rise (PALSEA): Ice-sheet and sea-level responses to past climate warming, Quat. Sc. Rev., 212, 28-32, https://doi.org/10.1016/j.quascirev.2019.03.032, 2019.

Carrassi, A., Bocquet, M., Bertino, L., and Evensen, G.: Data assimilation in the geosciences: An overview of methods, issues, and perspectives, WIREs Climate Change, 9, e535, https://doi.org/10.1002/wcc.535, 2018.

Düsterhus, A., Rovere, A., Carlson, A. E., Horton, B. P., Klemann, V., Tarasov, L., Barlow, N. L. M., Bradwell, T., Clark, J., Dutton, A., Gehrels, W. R., Hibbert, F. D., Hijma, M. P., Khan, N., Kopp, R. E., Sivan, D., and Törnqvist, T. E.: Palaeo-sea-level and palaeo-ice-sheet databases: problems, strategies, and perspectives, Clim. Past, 12, 911-921, https://doi.org/10.5194/cp-12-911-2016, 2016.

Dziewonski, A. M. and Anderson, D. L.: Preliminary Reference Earth Model, Phys. Earth Planet. In., 25, 297-356, https://doi.org/10.1016/0031-9201(81)90046-7, 1981.

Evensen, G.: Data assimilation: the ensemble Kalman filter, Springer Science \& Business Media, Berlin Heidelberg, 2nd edn., https://doi.org/10.1007/978-3-642-03711-5, 2009.

375 Farrell, W. E. and Clark, J. A.: On postglacial sea level, Geophys. J. Roy. Astr. S., 46, 647-667, https://doi.org/10.1111/j.1365246x.1976.tb01252.x, 1976.

Fearnhead, P.: Markov chain Monte Carlo, sufficient statistics, and particle filters, J. Comput. Graph. Stat., 11, 848-862, 2002.

Fearnhead, P. and Künsch, H. R.: Particle filters and data assimilation, Annu. Rev. Stat. Appl., 5, 421-449, https://doi.org/10.1146/annurevstatistics-031017-100232, 2018.

Fournier, A., Nerger, L., and Aubert, J.: An ensemble Kalman filter for the time-dependent analysis of the geomagnetic field, Geochem. Geophy. Geosy., 14, 4035-4043, https://doi.org/10.1002/ggge.20252, 2013.

Gordon, N. J., Salmond, D. J., and Smith, A. F. M.: Novel approach to nonlinear/non-Gaussian Bayesian state estimation, IEE Proc. F, 140, 107-113, https://doi.org/10.1049/ip-f-2.1993.0015, 1993.

Hagedoorn, J. M., Wolf, D., and Martinec, Z.: An estimate of global sea level rise inferred form tide gauge measurements using glacial isostatic models consistent with the relative sea level record, Pure Appl. Geophys., 164, 791-818, https://doi.org/10.1007/s00024-0070186-7, 2007.

Haskell, N.: The motion of a viscous fluid under a surface load, Physics, 6, 265-269, https://doi.org/10.1063/1.1745329, 1935.

Hibbert, F. D., Rohling, E. J., Dutton, A., Williams, F. H., Chutcharavan, P. M., Zhao, C., and Tamisiea, M. E.: Coral indicators of past sea-level change: A global repository of U-series dated benchmarks, Quat. Sc. Rev., 145, 1-56, https://doi.org/10.1016/j.quascirev.2016.04.019, 2016.

Imbrie, J., Boyle, E. A., Clemens, S. C., Duffy, A., Howard, W. R., Kukla, G., Kutzbach, J., Martinson, D. G., McIntyre, A., Mix, A. C., Molfino, B., Morley, J. J., Peterson, L. C., Pisias, N. G., Prell, W. L., Raymo, M. E., Shackleton, N. J., and Toggweiler, J. R.: On 
https://doi.org/10.5194/npg-2021-22

Preprint. Discussion started: 7 June 2021

(c) Author(s) 2021. CC BY 4.0 License.

(c) (i)

the Structure and Origin of Major Glaciation Cycles 1. Linear Responses to Milankovitch Forcing, Paleoceanography, 7, 701-738, https://doi.org/10.1029/92PA02253, 1992.

Irrgang, C., Saynisch, J., and Thomas, M.: Utilizing oceanic electromagnetic induction to constrain an ocean general circulation model: A data assimilation twin experiment, J. Adv. Model. Earth Sy., 9, 1703-1720, https://doi.org/10.1002/2017MS000951, 2017.

Kendall, R. A., Mitrovica, J. X., and Milne, G. A.: On post-glacial sea level - II. Numerical formulation and comparative results on spherically symmetric models, Geophys. J. Int., 161, 679-706, https://doi.org/10.1111/j.1365-246X.2005.02553.x, 2005.

Khan, N. S., Ashe, E., Shaw, T. A., Vacchi, M., Walker, J., Peltier, W. R., Kopp, R. E., and Horton, B. P.: Holocene Relative Sea-Level Changes from Near-, Intermediate-, and Far-Field Locations, Curr. Clim. Ch. Rep., 1, 247-262, https://doi.org/10.1007/s40641-015-0029-z, 2015.

Klemann, V., Martinec, Z., and Ivins, E. R.: Glacial isostasy and plate motion, J. Geodyn., 46, 95-103, https://doi.org/10.1016/j.jog.2008.04.005, 2008.

Lambeck, K., Smither, C., and Johnston, P.: Sea-level change, glacial rebound and mantle viscosity for northern Europe, Geophys. J. Int., 134, 102-144, https://doi.org/10.1046/j.1365-246x.1998.00541.x, 1998.

405 Lambeck, K., Purcell, A., Johnston, P., Nakada, M., and Yokoyama, Y.: Water-load definition in the glacio-hydro-isostatic sea-level equation, Quat. Sc. Rev., 22, 309-318, https://doi.org/10.1016/S0277-3791(02)00142-7, 2003.

Latinović, M.: A method for validation of GIA models using sea-level data with applications to Hudson Bay and SW Fennoscandia, Phd thesis, Free University, Berlin, 2021.

Liu, J. and West, M.: Combined parameter and state estimation in simulation-based filtering, in: Sequential Monte Carlo methods in practice, pp. 197-223, Springer, https://doi.org/10.1007/978-1-4757-3437-9_10, 2001.

Liu, J. S., Chen, R., and Logvinenko, T.: A theoretical framework for sequential importance sampling with resampling, in: Sequential Monte Carlo methods in practice, pp. 225-246, Springer, https://doi.org/10.1007/978-1-4757-3437-9_11, 2001.

Martinec, Z.: Spectral-finite element approach for three-dimensional viscoelastic relaxation in a spherical earth, Geophys. J. Int., 142, 117141, https://doi.org/10.1046/j.1365-246x.2000.00138.x, 2000.

415 Mitrovica, J. X. and Peltier, W. R.: The inference of mantle viscosity from an inversion of the Fennoscandian relaxation spectrum, Geophys. J. Int., 114, 45-62, https://doi.org/10.1111/j.1365-246x.1993.tb01465.x, 1993.

Nerger, L., Hiller, W., and Schröter, J.: PDAF - The parallel data assimilation framework: experiences with kalman filtering, in: Use of High Performance Computing in Meteorology, pp. 63-83, World Scientific, https://doi.org/10.1142/9789812701831_0006, 2005.

Peltier, W. R.: Mantle Viscosity and Ice-Age Ice Sheet Topography, Science, 273, 1359-1364, https://doi.org/10.1126/science.273.5280.1359, 1996.

Peltier, W. R.: Global Glacial Isostasy and the Surface of the Ice-Age Earth: The ICE-5G (VM 2) Model and GRACE, Annu. Rev. Earth Planet. Sci., 32, 111-149, https://doi.org/10.1146/annurev.earth.32.082503.144359, 2004.

Pham, D. T.: Stochastic methods for sequential data assimilation in strongly nonlinear systems, Mon. Weather Rev., https://doi.org/10.1175/1520-0493(2001)129<1194:SMFSDA>2.0.CO;2, 2001.

Poyiadjis, G., Doucet, A., and Singh, S. S.: Particle approximations of the score and observed information matrix in state space models with application to parameter estimation, Biometrika, 98, 65-80, https://doi.org/10.1093/biomet/asq062, 2011.

Rubin, D. B.: Using the SIR algorithm to simulate posterior distributions, Bayes. Stat., 3, 395-402, 1988.

Saynisch, J., Bergmann-Wolf, I., and Thomas, M.: Assimilation of GRACE-derived oceanic mass distributions with a global ocean circulation model, J. Geod., 89, 121-139, https://doi.org/10.1007/s00190-014-0766-0, 2015.

Shennan, I., Long, A. J., and Horton, B. P., eds.: Handbook of Sea-Level Research, Wiley, Blackwell, 2015. 
https://doi.org/10.5194/npg-2021-22

Preprint. Discussion started: 7 June 2021

(c) Author(s) 2021. CC BY 4.0 License.

(c) (1)

Steffen, H. and Wu, P.: Glacial isostatic adjustment in Fennoscandia-a review of data and modeling, J. Geodyn., 52, 169-204, https://doi.org/10.1016/j.jog.2011.03.002, 2011.

Unger, A., Schulte, S., Klemann, V., and Dransch, D.: A visual analysis concept for the validation of geoscientific simulation models, IEEE

T. Vis. Comput. Gr., 18, 2216-2225, https://doi.org/10.1109/tvcg.2012.190, 2012.

435 van de Plassche, O., ed.: Sea-Level Research: A Manual for the Collection and Evaluation of Data, Geo Books, Norwich, 1986.

Van der Merwe, R., Doucet, A., De Freitas, N., and Wan, E.: The Unscented Particle Filter, in: Advances in neural information processing systems, vol. 13, pp. 584-590, 2001.

Van Leeuwen, P. J.: Particle Filtering in Geophysical Systems, Mon. Weather Rev., pp. 4089-4114, https://doi.org/10.1175/2009MWR2835.1, 2009.

440 Wessel, P., Luis, J. F., Uieda, L., Scharroo, R., Wobbe, F., Smith, W. H. F., and Tian, D.: The Generic Mapping Tools Version 6, Geochem. Geophy. Geosy., 20, 5556-5564, https://doi.org/https://doi.org/10.1029/2019GC008515, 2019.

Whitehouse, P. L.: Glacial isostatic adjustment modelling: historical perspectives, recent advances, and future directions, Earth Surf. Dynam., 6, 401-429, https://doi.org/10.5194/esurf-6-401-2018, 2018. 